\title{
microRNA regulation of persistent stress-enhanced memory
}

\author{
Stephanie E. Sillivan ${ }^{1,2}$, Sarah Jamieson ${ }^{1,2}$, Laurence de Nijs $^{3}$, Meghan Jones ${ }^{1,2}$, Clara \\ Snijders $^{3}$, Torsten Klengel ${ }^{4}$, Nadine F. Joseph ${ }^{1,2}$, Julian Krauskop ${ }^{5}$, Jos Kleinjans ${ }^{5}$, \\ Christiaan H. Vinkers ${ }^{6}$, Marco P.M. Boks ${ }^{6}$, Elbert Geuze ${ }^{6,7}$, Eric Vermetten ${ }^{6,7,8}$, Sabina \\ Berretta $^{4}$, Kerry J. Ressler ${ }^{4}$, Bart P.F. Rutten ${ }^{3}$, Gavin Rumbaugh ${ }^{2}$, Courtney A. Miller ${ }^{1,2,{ }^{*}}$
}

${ }^{1}$ Department of Molecular Medicine, The Scripps Research Institute, Jupiter, FL USA ${ }^{2}$ Department of Neuroscience, The Scripps Research Institute, Jupiter, FL USA. ${ }^{3}$ School for Mental Health and Neuroscience, Department of Psychiatry and Neuropsychology, Maastricht University, Maastricht, The Netherlands. ${ }^{4}$ Department of Psychiatry, Harvard Medical School, McLean Hospital, Belmont, MA, USA. ${ }^{5}$ Department of Toxicogenomics, Maastricht University, Maastricht, The Netherlands. ${ }^{6}$ Brain Center Rudolf Magnus, Department of Psychiatry, University Medical Center Utrecht, The Netherlands. ${ }^{7}$ Research Centre for Military Mental Healthcare, Ministry of Defence, Utrecht, The Netherlands. ${ }^{8}$ Department of Psychiatry, Leiden University Medical Center, Leiden, The Netherlands.

\section{Abstract}

Disruption of persistent, stress-associated memories is relevant for treating posttraumatic stress disorder (PTSD) and related syndromes, which develop in a subset of individuals following a traumatic event. We previously developed a stress-enhanced fear learning (SEFL) paradigm in inbred mice that produces PTSD-like characteristics in a subset of mice, including persistently enhanced memory and heightened cFos in the basolateral amygdala complex (BLC) with retrieval of the remote (30 day old) stress memory. Here the contribution of BLC microRNAs (miRNAs) to stress-enhanced memory was investigated because of the molecular complexity they achieve through their ability to regulate multiple targets simultaneously. We performed small-RNA sequencing (smRNA-Seq) and quantitative proteomics on BLC tissue collected from mice one month after SEFL and identified persistently changed microRNAs, including mir-135b-5p, and proteins associated with PTSD-like heightened fear expression. Viral-mediated overexpression of mir-135b-5p in the BLC of stress-resilient animals enhanced remote fear memory expression and promoted spontaneous renewal 14 days after extinction. Conversely, inhibition of BLC mir-135b-5p in stress-susceptible animals had the opposite effect, promoting a resilient-like phenotype. mir-135b-5p is highly conserved across mammals and was detected in postmortem human amygdala, as well as human serum samples. The mir-135b passenger strand, mir-135b-3p, was significantly elevated in serum from PTSD military veterans, relative to combat-exposed control subjects. Thus, miR-135b-5p may be an important therapeutic target for dampening

Users may view, print, copy, and download text and data-mine the content in such documents, for the purposes of academic research, subject always to the full Conditions of use:http://www.nature.com/authors/editorial_policies/license.html\#terms

${ }^{*}$ Correspondence to: Courtney Miller cmiller@ scripps.edu, 130 Scripps Way, Jupiter, FL 33458, Phone 561-228-2958.

Disclosures: Authors declare no competing interests. 
persistent, stress-enhanced memory and its passenger strand a potential biomarker for responsivity to a mir-135-based therapeutic.

\section{Keywords}

resilience; susceptibility; PTSD; memory; biomarker

\section{Introduction}

Unlike memory formation, mechanisms supporting long-lasting, remote memory are largely unknown, yet highly relevant to psychiatric disorders marked by persistent, unwanted memories, such as posttraumatic stress disorder (PTSD). PTSD is a chronic, debilitating disorder in which patients exhibit heightened, perseverant and extinction-resistant memories of trauma ${ }^{1}$. Nearly everyone experiences at least one traumatic event, but only $10-20 \%$ display enduring symptoms of PTSD ${ }^{2}$. Exposure therapy, a form of cognitive behavioral therapy (CBT) that utilizes extinction of fearful memories, is considered the gold standard treatment. However, many patients are resistant or experience exacerbated symptoms with exposure therapy and, of those who respond, most retain their PTSD diagnosis ${ }^{3}$. Thus, development of adjunctive pharmacotherapies to enhance success of the various forms of CBT is needed. But first, deeper insight into the underlying neurobiology to identify potential targets is required. Specifically, there is a need to better understand the mechanisms that determine differential responses to stress and how those responses persist for extended periods of time.

To identify mechanisms governing persistent, differential stress susceptibility, we developed a stress-enhanced fear learning (SEFL) paradigm in inbred C57BL/6 mice that results in PTSD-like characteristics, including persistently enhanced memory, in a subset of mice termed stress susceptible (SS $)^{4}$. . This contrasts with stress resilient (SR) mice that do not display stress-induced enhancements. Importantly, the protocol allows for the study of molecular phenotypes associated with selective vulnerability because SS mice can be identified from training data, avoiding the mechanistic confounds introduced by the additional phenotyping commonly required. We also reported differential expression of genes with known polymorphisms in human PTSD genomic studies between SS and SR animals ${ }^{4}$.

A role for miRNAs in mediating memory has emerged over the past decade ${ }^{6}$. miRNAs are endogenous 20-24 nucleotide RNAs that act as translational repressors through direct binding to the 3'-UTR of target mRNAs and noncleavage degradation of target mRNA via deadenylation ${ }^{7}$. Protein translation is required for formation of new memories, and its regulation via synapse-specific miRNA localization can modulate synaptic plasticity ${ }^{8-10}$. Indeed, the expression of miRNA biogenesis regulators Dicer and Pasha influences fear memories ${ }^{11,12}$. Fear conditioning (FC) regulates miRNA expression and modulation of these miRNAs impacts consolidation and retrieval ${ }^{13-18}$. However, these prior studies all focused on recent, not remote, fear memory and the additional impact of stress is unknown. Yet, miRNAs are excellent remote memory candidate regulators because their wide genomic 
range of potential target proteins confers a complexity capable of handling the intricacies of memory. Importantly, mature miRNAs can have very long half-lives (i.e. months) ${ }^{19}$, lending themselves to the goal of memory persistence. Here we examined involvement of miRNAs in mediating susceptibility to remote, stress-enhanced memories in our SEFL paradigm, with the hypothesis that a unique miRNA signature defines susceptibility to stress-enhanced memory, differentiating it from stress resilience and providing a potential path for selective memory modulation 20,21 .

\section{Materials and Methods}

Animals:

Adult C57BL/6 mice, 8-10 weeks of age (The Jackson Laboratory, Bar Harbor, ME), were maintained on a 12:12 hour light/dark cycle and supplied with food and water ad libitum. Mice were group housed 3-4/cage, acclimated to the facility for 1 week then handled for 3 days prior to experiments. For additional details see Supplement 1.

\section{Behavior:}

Acute restraint stress and auditory fear conditioning (SEFL) were performed as previously described ${ }^{4}$. Extinction training consisted of 60 tone presentations over two days and retrieval consisted of 5 tones; both were performed in a context unique from training. Subthreshold training consisted of habituation to context A for $3 \times 4$ min trials 24 hours after Recall Test 1 , followed by 2 CS-US pairings at $0.15 \mathrm{~mA}$ the next day. 24 hours later, mice received Recall Test 2. For a full description of fear conditioning, extinction and anxiety tests (open field, elevated plus maze [EPM] and acoustic startle response), please refer to ${ }^{4}$ and Supplement 1.

\section{Human serum samples:}

Sequenced subjects $(\mathrm{N}=24)$ were part of a prospective cohort study of 1,032 Dutch military soldiers deployed to Afghanistan between 2005 and 2009 for four months ${ }^{22}$. All participants gave written informed consent and the study was conducted in accordance with the Declaration of Helsinki. For additional details see Supplement 1.

\section{Human brain tissue:}

Human amygdala containing all major sub-nuclei was dissected, snap frozen and stored at $-80^{\circ} \mathrm{C}$ until further processing. Tissue was isolated from four control post-mortem brain samples (details below) from the Harvard Brain and Tissue Resource Center (HBTRC) at Mclean Hospital.

\begin{tabular}{|c|c|c|c|c|c|l|}
\hline Sample & Group & Sex & Age & PMI & RIN & Cause of death \\
\hline$\# 30$ & Control & F & 44 & 10.7 & 7.8 & cardiac arrest \\
\hline$\# 8$ & Control & F & 61 & 16.8 & 6.7 & cardiopulmonary arrest/ myocardial infarction \\
\hline$\# 39$ & Control & M & 74 & 14.3 & 5.3 & cardiopulmonary arrest/ myocardial infarction \\
\hline$\# 55$ & Control & M & 57 & 17.3 & 5.1 & cardiac arrest; coronary artery disease \\
\hline
\end{tabular}

PMI: post-mortem interval in hours; RIN: RNA integrity number 
RNA extraction:

For rodent studies, total RNA was extracted from fresh frozen bilateral tissue punches using the miRVANA PARIS RNA extraction kit (Life Technologies, Carlsbad, CA). For human studies, total RNA was extracted using the Norgen RNA/DNA/Protein Purification Plus Kit (Norgen Biotek, Ontario, Canada) according to the manufacturer's protocol. For additional details, please see supplemental methods.

\section{miRNA Library Preparation, Sequencing and data analysis:}

Single end smRNA-seq was performed on mouse BLC samples using the TruSeq small RNA library prep kit (Illumina, San Diego, CA) or the NEBNext Small RNA Library Prep Set for Illumina (New England BioLabs Inc., Ipswich, MA, USA). miRNAs that had at least 0.5 $\log _{2}$ fold change between SS and SR in both sequencing runs were considered 'candidate miRNAs' and analyzed for pathway analysis. For human serum samples, libraries were also prepared with the TruSeq Kit For additional details, please see supplemental methods.

\section{QPCR:}

A cDNA library was created from 50ng of total RNA using the mirCURY LNA RT Kit (Qiagen, Germantown, MD) and PCR reactions were performed in triplicate for each sample using the miRCURY LNA SYBR Green PCR Kit and the following locked nucleic acid (LNA) SYBR green primers from Qiagen. Data were normalized using the $\Delta \Delta c_{t}$ method $^{23}$. Please see supplemental methods for additional details.

\section{Quantitative mass spectrometry:}

Pooled samples from each treatment group were submitted for liquid chromatography-mass spectrometry (LC-MS) at the Harvard Mass Spectrometry and Proteomics Resource Laboratory. Raw data were submitted for analysis in Proteome Discoverer 2.1.0.81 (Thermo Fisher) software. Candidate proteins were identified as those that had at least $1.5 \log _{2}$ fold change between treatment groups. Please see supplemental methods for additional details.

\section{miRNA inhibitors and lenti-miR viruses:}

Miridian miRNA hairpin inhibitors directed against mmu-miR-135b-5p or the nonmammalian miRNA cel-miR-67 were obtained from GE Dharmacon (Lafayette, CO). To overexpress (OE) mir-135b-5p, pre-miR-135b-5p or a scramble nontargeting control sequence in the lentiviral vector CD513 were obtained from System Biosciences (Palo Alto, $\mathrm{CA})$. The OE vector was packaged with GFP into lentivirus using a $2^{\text {nd }}$ generation packaging system at the Emory Viral Vector Core (Emory University, Atlanta, GA). Titers obtained were $3 \times 10^{8} \mathrm{iu} / \mathrm{ml}$ for $\mathrm{OE}$.

\section{Intra-amygdalar infusions:}

miRNA inhibitors, non-targeting controls, or lenti-mirs were injected bilaterally into the BLC (AP: $1.5 \mathrm{~mm}, \mathrm{ML}: \pm 3.2 \mathrm{~mm}$ from bregma and DV: $-4.7 \mathrm{~mm}$ from the skull) of mice as previously described ${ }^{20}$. Inhibitors were reconstituted in water then prepared with jetPEI transfection reagent (Polyplus Transfection, Illkirch, France). 1ul of 400ng/ul was injected 28 days after fear conditioning and then animals were tested 48 hours later. For lentiviral 
experiments, $1.5 \mathrm{ul}$ of viruses were injected 23 days after fear conditioning and animals were tested 1 week later to give sufficient time for viral expression.

\section{Dual fluorescence in situ hybridization (FISH):}

Expression analysis experiments to detect miRNAs were performed as described, with modification ${ }^{24}$. Please see supplemental methods for additional details.

\section{Cell fractionation:}

Fresh tissue was dissected from naïve animals and immediately placed into homogenization buffer as previously described ${ }^{25}$, with modification. Please see supplemental methods for additional details.

\section{Statistics and data analysis:}

All statistical results and sample sizes are reported in the figure legends. We identified candidate miRNAs based on fold change and reproducibility in two separate biological replicate cohorts of SEFL animals. miRNAs that had at least $0.5 \log _{2}$ fold change difference between SS and SR animals were considered candidate regulators of traumatic memory. For comparisons with more than one group, an ANOVA was performed with post-hoc Tukey ttests. For comparisons with two groups, t-test were performed. For behavioral analyses of datapoints across time or extinction bins, a repeated measures ANOVA was performed. For correlations of two datapoints, a Pearson correlation was performed with the R squared value reported.

\section{Results}

\section{Long-lasting dysregulation of miRNAs after stress-enhanced memory formation}

SEFL was performed in male mice, followed by a remote memory test 30 days later (Fig. 1A). As previously reported ${ }^{4}$, unbiased cluster analysis identifies varied memory strength at the recall test as a result of prior stress that was predicted by freezing during FC (Fig. 1B). Indeed, stressed animals that froze more during training displayed the highest freezing during the remote memory recall test and thus, were classified as SS. To interrogate mechanisms underlying differential memory strength between SS and SR animals, despite identical training and genetic homozygosity, we performed small RNA-sequencing (smRNA-Seq) and quantitative proteomics on basolateral amygdala complex (BLC; lateral and basolateral) tissue of SEFL-trained mice that did not undergo a retrieval test. Tissue was isolated 30 days after training to identify miRNAs in SS mice contributing to enhanced memory strength through persistent change following SEFL, not miRNA changes dynamically induced by the act of memory retrieval. The BLC was chosen because it is highly stress-responsive and fear memories rely on this region ${ }^{26}$. Further, we previously demonstrated that SS animals have exaggerated cFos activation in the BLC relative to SR and FC (no stress) animals, consistent with human PTSD imaging studies ${ }^{27}$. And, unlike hippocampus-dependent memories, for which influence shifts to the cortex as long-lasting memory develops, auditory fear memory continues to rely on the amygdala long after learning ${ }^{26}$. Importantly, freezing at the end of training, which classifies stress susceptibility, did not differ between SR and FC animals, but did differ between SR and SS (Fig. 1C). 
SmRNA-Seq was performed on two separate cohorts of animals at two genomics facilities employing different reagents and bioinformatics pipelines to yield miRNA profiles associated with susceptibility to stress-enhanced memory. We compared the two datasets and identified 42 miRNAs that were differentially expressed between SS and SR groups in both datasets (Fig. 1D, Supplemental Table 1 and Supplemental Excel Table.1-2). 41 of these were upregulated in SS animals. Pathway analysis with DIANA's mirPATH software using the microCts algorithm identified target pathways predicted to be regulated by these differentially expressed miRNAs. It included pathways that critically regulate stress and learning processes, including ECM-receptor interaction, MAPK signaling pathway and Thyroid Hormone Signaling (Fig. 1E). DIANA mirPath also provides access to two other target predicting algorithms, TargetScan and Tarbase. We compared all three algorithms and used the results to guide sequencing validation to miRNAs predicted by at least 2 algorithms that have targets significantly involved in pathways related to learning and memory (Supplemental Table 2).

One miRNA on this list, mir-135b-5p, is predicted to target proteins in both ECM-receptor interaction and Thyroid Hormone Signaling pathways. Interestingly, thyroid hormone signaling has been implicated in amygdala-based memory processes ${ }^{28,29}$ and family members of mir-135b-5p have been studied in depressive and anxiety-like behaviors ${ }^{30,31}$, as well as cortical axon guidance ${ }^{32}$ and synaptic plasticity ${ }^{33}$. Importantly, mir-135b-5p is conserved from mouse to human brain tissue ${ }^{34}$. Only one study has examined mir-135b-5p in the context of neuronal processes, identifying both mir-135a and mir-135b as regulators of axon guidance, migration and regeneration in cultured cortical and hippocampal cells ${ }^{32}$. We examined mir-135b-5p expression in the adult mouse brain and found it is broadly expressed in brain areas involved in emotion and memory, such as the BLC, the hypothalamus and the frontal cortex (Fig. 1F). In situ hybridization revealed anatomical localization of mir-135b-5p throughout the BLC (Fig. 1G). Using a cell fractionation assay, mir-135b-5p expression was detected throughout cells, including synaptosomes, where it could regulate synaptic protein translation underlying memory (Fig. 1H). Confirming the smRNA-Seq results by qPCR in an independent cohort that was not sequenced and did not undergo extinction training, we found BLC mir-135b-5p levels were elevated in SEFL animals that displayed the greatest freezing one month earlier, during training (Fig. 1I). The increase in mir-135b-5p was specific to the BLC, as no change was observed in other brain regions assessed from SEFL animals that have known roles in stress resilience and memory, such as the hippocampus, nucleus accumbens and the frontal cortex (Fig. 1J). Further, the selective elevation of levels of mir-135b-5p in SS BLC developed over time, as levels were equivalent across groups 24 hours and 7 days after SEFL (Fig. 1K). This suggests recruitment of mir-135b-5p during "incubation" of the fear memory ${ }^{35}$, rather than during its initial formation or consolidation. The validation of mir-135b-5p upregulation specifically in the BLC of SS animals 30 days after SEFL indicated that this candidate miRNA may contribute to the long-lasting traumatic memory phenotype. Therefore, we further explored the role of mir-135b-5p with in vivo functional manipulations in the SEFL model. 


\section{mir-135b-5p overexpression enhances memory expression in stress resilient mice}

We examined the functional role of mir-135b-5p in regulating persistent stress-enhanced memory using a lentivirus that overexpresses (OE) pre-mir-135b-5p (premirOEmir-135b-5p) or a scrambled non-targeting sequence to mimic the elevated mir-135b-5p seen in SS mice. We injected premirOE-135b-5p into the BLC of SR mice 3 weeks after SEFL and performed extinction one week later (Fig. 2A). Enhanced memory expression was observed in SR-premirOE-135b-5p mice (Fig. 2B-C). Extinction was achieved with a strong protocol (Supplemental Fig. 1), yet mir-135b-5p levels remained elevated at the conclusion of extinction in SR-premirOE-135b-5p mice relative to SR-nontargeting control animals (Fig. 2D), suggesting the potential for spontaneous recovery if mediated by mir-135b-5p. Indeed, a 5-tone recall test two weeks after extinction demonstrated a return of enhanced fear memory in SR-premirOE-135b-5p animals (Fig. 2E). Anxiety-related open field, elevated plus maze and acoustic startle behaviors were no different in naïve animals injected with premirOE-135b-5p in the BLC or the nontargeting control, indicating that OE of mir-135b-5p is not simply anxiogenic (Supplemental Fig. S2).

\section{mir-135b-5p inhibition selectively ameliorates stress-enhanced memory expression in stress susceptible mice}

To explore the converse functional impact of mir-135b-5p on stress-enhanced memory, we utilized a synthetic hairpin mir-135b-5p inhibitor (INH) that sequesters mir-135b-5p to assess the consequence of mir-135b-5p inhibition on stress-enhanced memory in SS mice. We injected the INH into the BLC of mice 28 days after SEFL, followed by extinction 2 days later (Fig. 3A). INH-mir-135b-5p reduced freezing in SS mice relative to a nontargeting control inhibitor during the first $10 \mathrm{CS}+$ tone presentations (Fig. 3B-C). In addition, consistent with mir-135b-5p's role in memory enhancement, the miRNA appeared to have been degraded during the extinction process in the INH-mir-135b-5p animals, which displayed reduced freezing, as its levels were markedly reduced in the INH-mir-135b-5p group by the end of the extinction protocol ( 2 days; Fig. 3D). Two weeks after the two day extinction protocol, which results in complete extinction (Supplemental Fig. 3), we assessed extinction retention with a 5-tone recall test and observed no differences between the groups (Fig. 3E). However, a subthreshold training protocol resulted in further enhancement of the fear memory in SS-INH-nontargeting controls that was absent in SS-INH-mir-135b-5p mice, suggesting a persistent dampening of memory via prior mir-135b-5p inhibition, rather than acute suppression of retrieval (Fig. 3F).

Interestingly, further knockdown of BLC mir-135b-5p in SR animals via BLC infusion of INH-mir-135b-5p did not alter memory (Fig. 3G). This may not be surprising, as levels of mir-135b-5p are not different between FC and SR males (Fig. 1I). We have previously reported that SEFL females do not cluster into stress-induced subgroups, but rather, display a more uniform SS-like phenotype ${ }^{4}$. Here we find that levels of mir-135b-5p do not differ between FC and SEFL females, regardless of their freezing during training (Fig. 3H). In accordance with this, INH-mir-135b-5p in SEFL females does not reduce memory strength (Fig. 3I). Together this suggests not only a sex-specific role for mir-135b-5p, but also a threshold of its expression must be reached to achieve pathologic memory effects and subsequent amelioration by reduction of mir-135b-5p levels. Further, BLC mir-135b-5p 
levels are not merely anxiotropic, as we observed no differences in anxiety-related open field and elevated plus maze tasks after INH-mir-135b-5p infusion in naïve mice (Supplemental Fig. 2). Importantly, delivery of non-targeting viral and inhibitor controls to the BLC did not disrupt the expected phenotypic differences in memory strength between SS and SR animals ${ }^{4}$ (Supplemental Fig. 2), indicating that behavioral differences observed also cannot be attributed to technical manipulation of the BLC disrupting stress-associated phenotypes.

\section{mir-135b is conserved in humans and elevated in serum of military personnel with PTSD}

The mir-135b stem loop sequence is $100 \%$ conserved from mouse to human (Fig. 4A). While amygdala tissue from human PTSD subjects could not be obtained for this project, we measured levels of mature mir-135b-5p and the $-3 p$ arm, indicated to be the passenger strand by miRBase 22, in postmortem human amygdala tissue by qPCR (Fig. 4B and Supplemental Fig. 4). Both were detected, but $-5 \mathrm{p}$ was expressed at much higher levels than $-3 p$, indicating this is the major mir-135b isoform in the human amygdala. This is consistent with miRBase 22's assignment of $-3 p$ as the passenger strand and our own finding that $-3 p$ levels in the mouse BLC and plasma are below the level of detection by qPCR. Interestingly, $-5 p$ levels are also below the level of detection in mouse plasma. We next measured levels of mir-135b-5p and $-3 p$ in serum samples collected from a cohort of well-characterized male Dutch military six months after a four month deployment in Afghanistan, at which time trauma exposure and PTSD symptomatology were assessed. mir-135b-3p was selectively elevated in members of the military diagnosed with PTSD ("susceptible") relative to members without the diagnosis ("resilient") and non-trauma exposed healthy military controls (Fig. 4C-D and Supplemental Excel Table 3). Consistent with levels of $-3 p$ being low, there were two samples with values of 0 in the data, one in the Control group and one in the PTSD Resilient group. To ensure that these two data points were not responsible for the significant elevation in the PTSD Susceptible group, we first confirmed they were not outliers by a Grubbs Test. They could not be statistically excluded based on a Grubbs test, but we still repeated the ANOVA without the data points and found that the statistical support for selective elevation of mir-135b-3p in PTSD Susceptible was further strengthened by their exclusion $\left(\mathrm{F}_{(2,15)}=18.65, \mathrm{P}<0.0001\right.$; Post hoc: Control vs PTSD Susceptible $\mathrm{P}=0.0002$, PTSD Resilient vs Susceptible $\mathrm{P}=0.0012$ ). Passenger strands have traditionally been thought to be degraded upon release from the mature strand at the site of production. However, growing evidence indicates they can enter circulation and functionally impact distal structures ${ }^{36}$. Therefore, it will be important to identify the sites and actions of elevated mir-135b-3p. Regardless, this identifies mir-135b-3p's potential as a biomarker of PTSD.

\section{Long-lasting BLC proteomic dysregulation in SS mice includes mir-135b-5p putative targets}

While some studies on miRNAs in the brain have successfully identified an individual protein target capable of recapitulating the behavioral effect of the miRNA of interest, these protein targets often have a wide genomic impact themselves. Examples include transcription factors and epigenetic modifiers ${ }^{37,38}$. However, one appealing aspect of miRNAs in the context of psychiatric disorders is the potential for a single miRNA to simultaneously titrate the levels of multiple protein targets to achieve behavioral outcomes. Therefore, we performed quantitative mass spectrometry (MS) on BLC tissue from SEFL 
animals 30 days after training (no extinction) to identify proteome-wide potential mir-135b-5p targets that may participate in stress-enhanced memory. 3,560 proteins were detected in the BLC by MS, with 72 downregulated and 663 upregulated by at $>1.5 \log _{2}$ fold change between SS and SR (Fig. 5A and Supplemental Excel Table 4). The top 10 most significant pathways from Ingenuity Pathway Analysis (IPA) revealed functional annotations related to neuronal structure (e.g. Density of microtubules, Organization of cytoskeleton) in the downregulated protein list (Fig. 5B), while the upregulated pathways included transport of molecules and several related to cancers (Fig. 5C). The 5 proteins with the greatest increase in SS BLC are listed in Figure 5D. While many proteins are expected to participate in miRNA-independent processes, downregulation of SS proteins could reflect a coordinated interaction with upregulated miRNAs that are responsive to stress-enhanced memory, such as mir-135b-5p. A total of 272 mir-135b-5p targets were predicted by at least two of the three major databases (TargetScan, DIANA, mirDB), but only 76 were detected in BLC tissue at both the RNA and protein level (Fig. 5E). Interestingly, 24\% (18) of predicted mir-135b-5p protein targets detected in the BLC were regulated between SS and SR animals in the proteomics dataset and included Ntrk2, the TrkB BDNF receptor well characterized for its role in amygdala-dependent fear and extinction learning, as well as PTSD (Fig. 5EF) ${ }^{39}$. Pathway analysis of these 18 mir-135b-5p putative targets that were changed by SEFL identified annotations related to neuronal function, including spatial learning, long-term depression of neurons, plasticity of synapse and excitatory postsynaptic potential of neurons (Fig. 5G). Because many predicted mir-135b-5p targets could be false positives, we also examined the BLC proteome of naïve animals injected with premirOE-135b-5p in the BLC at physiologically relevant levels. We aligned the MS datasets and identified 13 proteins downregulated by $>50 \%$ in both SS animals and naive premirOE-135b-5p animals (Fig. $5 \mathrm{H}$ ), with functions including protein folding and degradation (Bag3), chromosome looping (Hmgb2), ion channel trafficking (Caskin1) and vesicle transport (Arfgap1). The number of potential targets increased to 99 proteins with a criteria of downregulation by $>25 \%$. IPA identified pathways related to neuronal function (e.g. Neuromuscular disease, Disorder of basal ganglia), as well as non-neuronal pathways with molecules in need of future functional studies in the brain (Fig. 5I).

\section{Discussion}

Utilizing a stress-enhanced fear learning protocol we developed ${ }^{4}$, that results in differential stress susceptibility in an inbred mouse strain commonly used in neuroscience, c57bl/6, we identified 42 miRNAs differentially expressed in the amygdala between susceptible and resilient mice one month after training. Based on its conservation in humans and predicted regulation of processes that could be reasonably expected to contribute to memory, we selected one of these, mir-135b-5p, for in-depth study. After confirming that remote (between 7 and 30 days post-SEFL) upregulation of mir-135b-5p is selective to SS mice, we further determined the change was unique to the amygdala, in that mir-135b-5p levels were unchanged by SEFL in other memory and stress-related brain regions. Elevating local BLC levels of mir-135b-5p in resilient mice enhanced remote fear memory expression, phenocopying the stress-enhanced memory displayed by SS mice, and resulted in spontaneous renewal of the memory two weeks after complete extinction. Conversely, intra- 
amygdala inhibition of mir-135b-5p in SS mice resulted in a marked reduction in freezing and suppressed renewal of the memory with subthreshold training two weeks after complete extinction.

The bidirectional impact of mir-135b-5p manipulation on memory strength begs the question of what memory process is most likely being targeted. miRNA levels were measured by smRNA-seq one month after SEFL training in the absence of retrieval, in order to identify contributors to enhanced remote memory strength observed in SS mice, as opposed to dynamic, retrieval-induced changes. Our hypothesis that mir-135b-5p contributes to the long-term storage of remote, stress-enhanced memory will require development of a small molecule inhibitor in order to achieve tight temporal control of the miRNA's activity ${ }^{40}$. However, some evidence in support of the memory storage hypothesis can be drawn from the present results. First, mir-135b-5p's elevation associated with stress susceptibility is present in SS mice at rest in the home cage, rather than being retrievalinduced. mir-135b-5p's impact on memory could still be due to enhanced retrieval of remote fear memories in SS mice. However, prior inhibition of mir-135b-5p in SS mice prevented the return of fear after subthreshold training two weeks later, indicating this miRNA has a role beyond the acute blockade of retrieval. In addition, the lack of an effect of mir-135b-5p inhibition in SR males and SEFL females argues against INH-mir-135b-5p producing a retrieval deficit. Accelerated extinction is also an unlikely explanation for the reduction in fear memory strength in SS mice following knockdown of mir-135b-5p, as the inhibitor, delivered 48 hours prior, had an immediate effect on memory expression, present from the first conditioned stimulus presentation. A blockade of reconsolidation is also unlikely, as the reduction in memory strength in INH-mir-135b-5p SS mice was not retrieval-dependent; reduced freezing was present from the start of the first retrieval test. Taken together, the most parsimonious explanation is that mir-135b-5p specifically contributes to the storage of stress-enhanced fear memory within the amygdala. While a pharmacological approach will be needed to definitively address this, we are intrigued by the possibility that maladaptive, stress-enhanced memory could be selectively titrated, without influencing other memories, as suggested by the results presented in Figure 3. Indeed, there is precedent for the selective, retrieval-independent targeting of maladaptive memories, as we have previously identified a mechanism unique to the storage of methamphetamine-associated memory that allows for its selective disruption ${ }^{20,21}$.

Combining smRNA-Seq with global quantitative MS identified a number of putative miRNA-dependent and -independent pathways differentially regulated between SS and SR animals that warrant further investigation, as many are likely critical regulators of the maladaptive behavioral and neurochemical adaptations associated with persistent, stressenhanced memory. This approach is also powerful, in that target identification is not limited to the miRNA target prediction algorithms, which are unable to take into account factors such as tissue and cell type-specificity and noncanonical miRNA-target interactions, such as center binding. Indeed, without this a priori prediction filter, our quantitative MS identified many proteins regulated in the context of mir-135b-5p overexpression that are not predicted mir-135b-5p targets by Targetscan. These changes are likely a combination of secondary responses to the overexpression and true, direct mir-135b-5p mediated suppression that warrant additional investigation. These include CASK Interacting Protein 1 (Caskin1), a 
cytosolic protein that binds scaffolding membrane proteins such as CASK at presynaptic sites $^{41}$; ADP Ribosylation Factor GTPase Activating Protein 1 (Arfgap1), a GTPase that regulates vesicle release and protein trafficking within the Golgi and endoplasmic reticulum $^{42}$; and TANK Binding Kinase 1 (Tbk1), a serine/threonine kinase for which variants have been associated with early-onset Alzheimer disease ${ }^{43}$. It will be interesting to determine if disruption of a single mir-135b-5p target is sufficient to recapitulate the impact of inhibiting the miRNA itself, or if the simultaneous inhibition of several targets, a unique capability of miRNAs, is required.

Further bolstering the therapeutic potential of mir-135b-5p, we found that this conserved miRNA is expressed in human amygdala and the miRNA's passenger strand, mir-135b-3p, is elevated in individuals from a well-characterized military cohort diagnosed with PTSD after a four month deployment to Afghanistan. The upregulation of mir-135b-3p was specific to susceptible individuals, as it was not elevated in individuals from the same military cohort that did not develop PTSD as a result of the same combat exposure. In Figure 3 we report that the elevation of mir-135b-5p is specific to male mice, as is the behavioral impact of reducing intra-amygdala levels of the miRNA. Interestingly, the Dutch military cohort consisted solely of men. Thus, it will be important to ascertain the levels of mir-135b in females diagnosed with PTSD in order to determine the extent to which this miRNA's impact is sex-specific. Regardless, the correspondence between the SEFL findings and results in humans with a PTSD diagnosis argues for the therapeutic discovery potential of the SEFL protocol ${ }^{4}$ and suggests that mir-135b-3p may serve as a biomarker capable of identifying individuals that would be responsive to a mir-135b-based therapeutic ${ }^{40}$.

\section{Supplementary Material}

Refer to Web version on PubMed Central for supplementary material.

\section{Acknowledgements}

We thank the Scripps Florida Genomics Core for sequencing services, Nripesh Prasad at the Genomic Services Lab at Hudson Alpha for sequencing services and data analysis, Adrian Reich and the Bioinformatics Core for data analysis, the Mouse Behavior core and Alicia Brantley for assistance and behavioral equipment, all members of the Miller/Rumbaugh Labs for their technical assistance and thoughtful discussions. This work was funded by grants from the National Institute of Mental Health MH105400 and MH105400-02 (Diversity Supplement) (CM), National Institute of Neurological Disorders and Stroke NS096833 (CM), National Institute on Drug Abuse DA041469 (SS) and the Brain and Behavior Foundation-NARSAD Young Investigator Award (SS). This research project was supported in part by the Viral Vector Core of the Emory Neuroscience National Institute of Neurological Disorders and Stroke Core Facilities grant, P30NS055077. LdN and smRNA-Seq experiments in human serum were funded by a VIDI award number 91718336 from the Netherlands Scientific Organization (BR) and the European Union's Horizon 2020 research and innovation programme under the Marie Skłodowska-Curie grant agreement No 707362 (LDN).

\section{References:}

1. American Psychiatric Association. Diagnostic and Statistical Manual of Mental Disorders. 5th edn: Washington, DC, 2013.

2. Kessler RC, Chiu WT, Demler O, Merikangas KR, Walters EE. Prevalence, severity, and comorbidity of 12-month DSM-IV disorders in the National Comorbidity Survey Replication. Arch Gen Psychiatry 2005; 62(6): 617-627. [PubMed: 15939839] 
3. Schottenbauer MA, Glass CR, Arnkoff DB, Tendick V, Gray SH. Nonresponse and dropout rates in outcome studies on PTSD: review and methodological considerations. Psychiatry 2008; 71(2): 134 168. [PubMed: 18573035]

4. Sillivan SE, Joseph NF, Jamieson S, King ML, Chevere-Torres I, Fuentes I et al. Susceptibility and Resilience to Posttraumatic Stress Disorder-like Behaviors in Inbred Mice. Biol Psychiatry 2017; 82(12): 924-933. [PubMed: 28778658]

5. Levinsohn EA, Ross DA. To Bend and Not Break: The Neurobiology of Stress, Resilience, and Recovery. Biol Psychiatry 2017; 82(12): e89-e90. [PubMed: 29129201]

6. Bredy TW, Lin Q, Wei W, Baker-Andresen D, Mattick JS. MicroRNA regulation of neural plasticity and memory. Neurobiol Learn Mem 2011; 96(1): 89-94. [PubMed: 21524708]

7. Eulalio A, Huntzinger E, Nishihara T, Rehwinkel J, Fauser M, Izaurralde E. Deadenylation is a widespread effect of miRNA regulation. RNA 2009; 15(1): 21-32. [PubMed: 19029310]

8. Hou Q, Ruan H, Gilbert J, Wang G, Ma Q, Yao WD et al. MicroRNA miR124 is required for the expression of homeostatic synaptic plasticity. Nat Commun 2015; 6: 10045. [PubMed: 26620774]

9. Lee K, Kim JH, Kwon OB, An K, Ryu J, Cho K et al. An activity-regulated microRNA, miR-188, controls dendritic plasticity and synaptic transmission by downregulating neuropilin-2. J Neurosci 2012; 32(16): 5678-5687. [PubMed: 22514329]

10. Schratt GM, Tuebing F, Nigh EA, Kane CG, Sabatini ME, Kiebler M et al. A brain-specific microRNA regulates dendritic spine development. Nature 2006; 439(7074): 283-289. [PubMed: 16421561]

11. Fiorenza A, Lopez-Atalaya JP, Rovira V, Scandaglia M, Geijo-Barrientos E, Barco A. Blocking miRNA Biogenesis in Adult Forebrain Neurons Enhances Seizure Susceptibility, Fear Memory, and Food Intake by Increasing Neuronal Responsiveness. Cereb Cortex 2016; 26(4): 1619-1633. [PubMed: 25595182]

12. Konopka W, Kiryk A, Novak M, Herwerth M, Parkitna JR, Wawrzyniak M et al. MicroRNA loss enhances learning and memory in mice. J Neurosci 2010; 30(44): 14835-14842. [PubMed: 21048142]

13. Dias BG, Goodman JV, Ahluwalia R, Easton AE, Andero R, Ressler KJ. Amygdala-dependent fear memory consolidation via miR-34a and Notch signaling. Neuron 2014; 83(4): 906-918. [PubMed: 25123309]

14. Volk N, Paul ED, Haramati S, Eitan C, Fields BK, Zwang R et al. MicroRNA-19b associates with Ago2 in the amygdala following chronic stress and regulates the adrenergic receptor beta 1 . $\mathrm{J}$ Neurosci 2014; 34(45): 15070-15082. [PubMed: 25378171]

15. Griggs EM, Young EJ, Rumbaugh G, Miller CA. MicroRNA-182 regulates amygdala-dependent memory formation. J Neurosci 2013; 33(4): 1734-1740. [PubMed: 23345246]

16. Lin Q, Wei W, Coelho CM, Li X, Baker-Andresen D, Dudley K et al. The brain-specific microRNA miR-128b regulates the formation of fear-extinction memory. Nat Neurosci 2011; 14(9): 1115-1117. [PubMed: 21841775]

17. Mathew RS, Tatarakis A, Rudenko A, Johnson-Venkatesh EM, Yang YJ, Murphy EA et al. A microRNA negative feedback loop downregulates vesicle transport and inhibits fear memory. Elife 2016; 5.

18. Wang RY, Phang RZ, Hsu PH, Wang WH, Huang HT, Liu IY. In vivo knockdown of hippocampal miR-132 expression impairs memory acquisition of trace fear conditioning. Hippocampus 2013; 23(7): 625-633. [PubMed: 23520022]

19. Schaefer A, O'Carroll D, Tan CL, Hillman D, Sugimori M, Llinas R et al. Cerebellar neurodegeneration in the absence of microRNAs. J Exp Med 2007; 204(7): 1553-1558. [PubMed: 17606634]

20. Young EJ, Blouin AM, Briggs SB, Sillivan SE, Lin L, Cameron MD et al. Nonmuscle myosin IIB as a therapeutic target for the prevention of relapse to methamphetamine use. Mol Psychiatry 2016; 21(5): 615-623. [PubMed: 26239291]

21. Young EJ, Aceti M, Griggs EM, Fuchs RA, Zigmond Z, Rumbaugh G et al. Selective, retrievalindependent disruption of methamphetamine-associated memory by actin depolymerization. Biol Psychiatry 2014; 75(2): 96-104. [PubMed: 24012327] 
22. Rutten BPF, Vermetten E, Vinkers CH, Ursini G, Daskalakis NP, Pishva E et al. Longitudinal analyses of the DNA methylome in deployed military servicemen identify susceptibility loci for post-traumatic stress disorder. Mol Psychiatry 2018; 23(5): 1145-1156. [PubMed: 28630453]

23. Livak KJ, Schmittgen TD. Analysis of relative gene expression data using real-time quantitative PCR and the 2(-Delta Delta C(T)) Method. Methods 2001; 25(4): 402-408. [PubMed: 11846609]

24. Kasai A, Kakihara S, Miura H, Okada R, Hayata-Takano A, Hazama K et al. Double In situ Hybridization for MicroRNAs and mRNAs in Brain Tissues. Front Mol Neurosci 2016; 9: 126. [PubMed: 27920667]

25. Most D, Ferguson L, Blednov Y, Mayfield RD, Harris RA. The synaptoneurosome transcriptome: a model for profiling the emolecular effects of alcohol. Pharmacogenomics J 2015; 15(2): 177-188. [PubMed: 25135349]

26. Gale GD, Anagnostaras SG, Godsil BP, Mitchell S, Nozawa T, Sage JR et al. Role of the basolateral amygdala in the storage of fear memories across the adult lifetime of rats. J Neurosci 2004; 24(15): 3810-3815. [PubMed: 15084662]

27. Stevens JS, Kim YJ, Galatzer-Levy IR, Reddy R, Ely TD, Nemeroff CB et al. Amygdala Reactivity and Anterior Cingulate Habituation Predict Posttraumatic Stress Disorder Symptom Maintenance After Acute Civilian Trauma. Biol Psychiatry 2017; 81(12): 1023-1029. [PubMed: 28117048]

28. Barez-Lopez S, Montero-Pedrazuela A, Bosch-Garcia D, Venero C, Guadano-Ferraz A. Increased anxiety and fear memory in adult mice lacking type 2 deiodinase. Psychoneuroendocrinology 2017; 84: 51-60. [PubMed: 28654773]

29. Montero-Pedrazuela A, Fernandez-Lamo I, Alieva M, Pereda-Perez I, Venero C, Guadano-Ferraz A. Adult-onset hypothyroidism enhances fear memory and upregulates mineralocorticoid and glucocorticoid receptors in the amygdala. PLoS One 2011; 6(10): e26582. [PubMed: 22039511]

30. Issler O, Haramati S, Paul ED, Maeno H, Navon I, Zwang R et al. MicroRNA 135 is essential for chronic stress resiliency, antidepressant efficacy, and intact serotonergic activity. Neuron 2014; 83(2): 344-360. [PubMed: 24952960]

31. Mannironi C, Biundo A, Rajendran S, De Vito F, Saba L, Caioli S et al. miR-135a Regulates Synaptic Transmission and Anxiety-Like Behavior in Amygdala. Mol Neurobiol 2017.

32. van Battum EY, Verhagen MG, Vangoor VR, Fujita Y, Derijck A, O’Duibhir E et al. An ImageBased miRNA screen identifies miRNA-135s as regulators of CNS axon growth and regeneration by targeting Kruppel-like factor 4. J Neurosci 2017.

33. Hu HY, Guo S, Xi J, Yan Z, Fu N, Zhang X et al. MicroRNA expression and regulation in human, chimpanzee, and macaque brains. PLoS Genet 2011; 7(10): e1002327. [PubMed: 22022286]

34. de Rie D, Abugessaisa I, Alam T, Arner E, Arner P, Ashoor H et al. An integrated expression atlas of miRNAs and their promoters in human and mouse. Nat Biotechnol 2017; 35(9): 872-878. [PubMed: 28829439]

35. Pickens CL, Golden SA, Adams-Deutsch T, Nair SG, Shaham Y. Long-lasting incubation of conditioned fear in rats. Biol Psychiatry 2009; 65(10): 881-886. [PubMed: 19167702]

36. Wu CaA P MicroRNA Passenger Strand. Circulation Cardiovascular Genetics 2014; 7: 567-568. [PubMed: 25140065]

37. Hollander JA, Im HI, Amelio AL, Kocerha J, Bali P, Lu Q et al. Striatal microRNA controls cocaine intake through CREB signalling. Nature 2010; 466(7303): 197-202. [PubMed: 20613834]

38. Im HI, Hollander JA, Bali P, Kenny PJ. MeCP2 controls BDNF expression and cocaine intake through homeostatic interactions with microRNA-212. Nat Neurosci 2010; 13(9): 1120-1127. [PubMed: 20711185]

39. Andero R, Ressler KJ. Fear extinction and BDNF: translating animal models of PTSD to the clinic. Genes Brain Behav 2012; 11(5): 503-512. [PubMed: 22530815]

40. Velagapudi SP, Gallo SM, Disney MD. Sequence-based design of bioactive small molecules that target precursor microRNAs. Nat Chem Biol 2014; 10(4): 291-297. [PubMed: 24509821]

41. Stafford RL, Ear J, Knight MJ, Bowie JU. The molecular basis of the Caskin1 and Mint1 interaction with CASK. J Mol Biol 2011; 412(1): 3-13. [PubMed: 21763699]

42. Chornyy S, Parnis A, Shmoish M, Cassel D. High abundance of ArfGAP1 found in the mossy fibers in hilus of the dentate gyrus region of the mouse brain. PLoS One 2017; 12(12): e0189659. [PubMed: 29240824] 
43. Verheijen J, van der Zee J, Gijselinck I, Van den Bossche T, Dillen L, Heeman B et al. Common and rare TBK1 variants in early-onset Alzheimer disease in a European cohort. Neurobiol Aging 2018; 62: 245.e241-245.e247. 

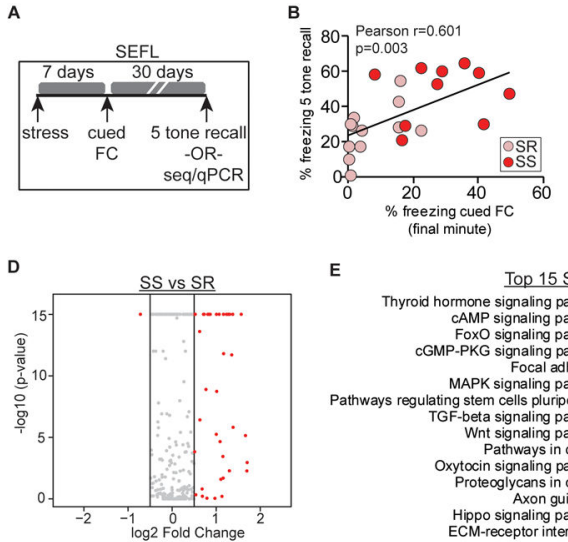

E

E
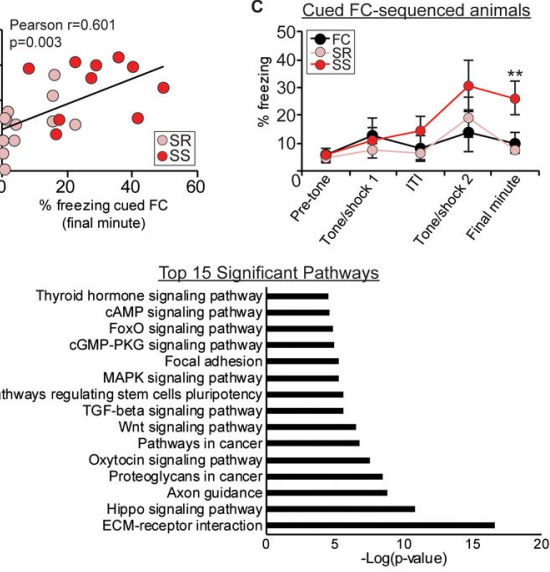
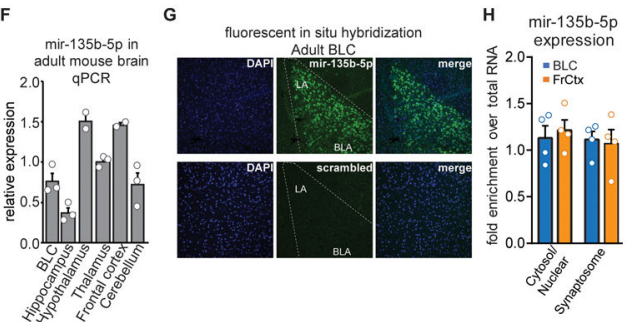

Figure 1. SEFL induces long-lasting miRNA changes in the BLC

(A) Overview of experimental paradigm to study long-term memory storage in SEFL mice.

(B) Pearson correlation between freezing during 5-tone recall test and freezing during cued FC in SEFL trained mice, with animals divided into SR (pink) or SS (red) subgroups based on training performance ${ }^{4}, r=0.601, p=0.003, n=22$. (C) Cued FC profile of SEFL trained animals used for smRNA-seq (no retrieval). RM-ANOVA between SR and SS: $\mathrm{F}_{(1,14)}=$ 5.05; $\mathrm{p}=0.041$; post hoc $\mathrm{t}$-test: $\mathrm{t}_{(14)}=3.315, \mathrm{p}=0.0005$. $\mathrm{n}=6-9 /$ group. (D) Volcano plot of miRNA expression in SS vs SR animals. (E) Pathway analysis of miRNAs listed in D using DIANA mirPath software and the microCts algorithm to identify pathways significantly targeted by SS miRNAs. (F) Characterization of mir-135b-5p expression in the adult mouse brain, $\mathrm{n}=2-3 /$ region. (G) Localization of mir-135b-5p in the BLC measured with in situ hybridization. (H) Characterization of mir-135b-5p expression in subcellular fractions to determine localization, $n=4 /$ region. (I) qPCR validation of elevated BLC mir-135b-5p expression in SS mice. One-way ANOVA: $\mathrm{F}_{(3,12)}=5.532 ; \mathrm{p}=0.0128$; Post-hoc Tukey test $\mathrm{p}<0.05$ for high vs FC $(*)$ and high vs moderate (\#), $\mathrm{n}=4-7 /$ group. (J) mir-135b-5p levels in the hippocampus hippocampus (Hpc), nucleus accumbens (NAc) or the frontal cortex (FrCtx) of mice 30 days after SEFL (no extinction), $\mathrm{n}=8-12$ /group. (K) mir-135b-5p levels in the BLC 24 hours or 7 days after SEFL (no extinction), n=6-8/group. Error bars are \pm S.E.M. 

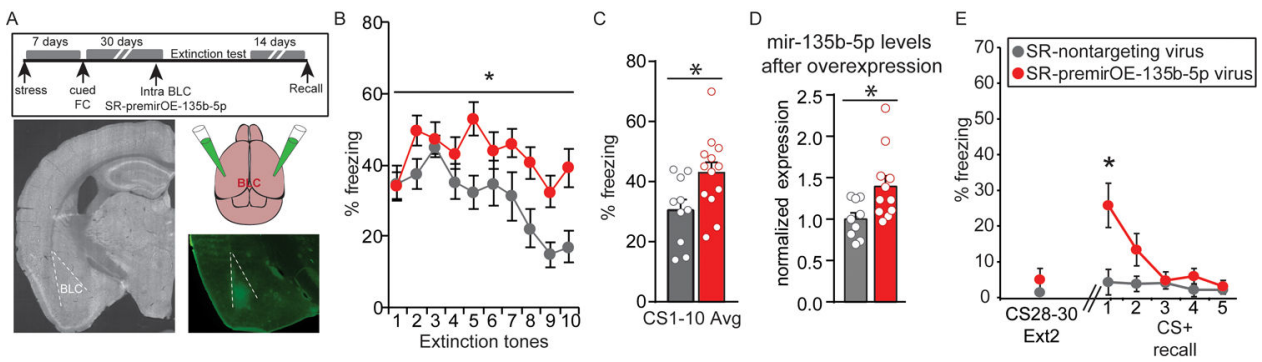

Figure 2. mir-135b-5p overexpression enhances traumatic memory expression in SR mice. (A) Overview of strategy to OE BLC mir-135b-5p in SR animals. (B-C) Freezing during the first ten tones of extinction on Day 1. RM-ANOVA for 10 tones extinction Day 1: $\mathrm{F}_{(1,21)}=$ 6.04; $\mathrm{p}=0.023$. Unpaired t-test for average of 10 tones: $\mathrm{t}_{(21)}=2.457, \mathrm{p}=0.0228$.

Nontargeting virus $n=10$, premirOE-135b-5p $n=13$. (D) mir-135b-5p levels in SR mice after extinction. Unpaired t-test: $\mathrm{t}_{(18)}=2.532, \mathrm{p}=0.0209$. Nontargeting virus $\mathrm{n}=9$; premirOE-135b-5p $n=11$. (E) Extinction retention from the end of extinction day 2 (average freezing to tones 28-30) to a 5 tone recall test 2 weeks later. RM-ANOVA 5 tone recall: $\mathrm{F}_{(1,20)}=5.27, \mathrm{p}=0.033$. Post-hoc t-test for tone $1: \mathrm{t}_{(20)}=2.673, \mathrm{p}=0.0146$. Nontargeting virus $n=9$, premirOE-135b-5p $n=13$. Error bars are \pm S.E.M. 


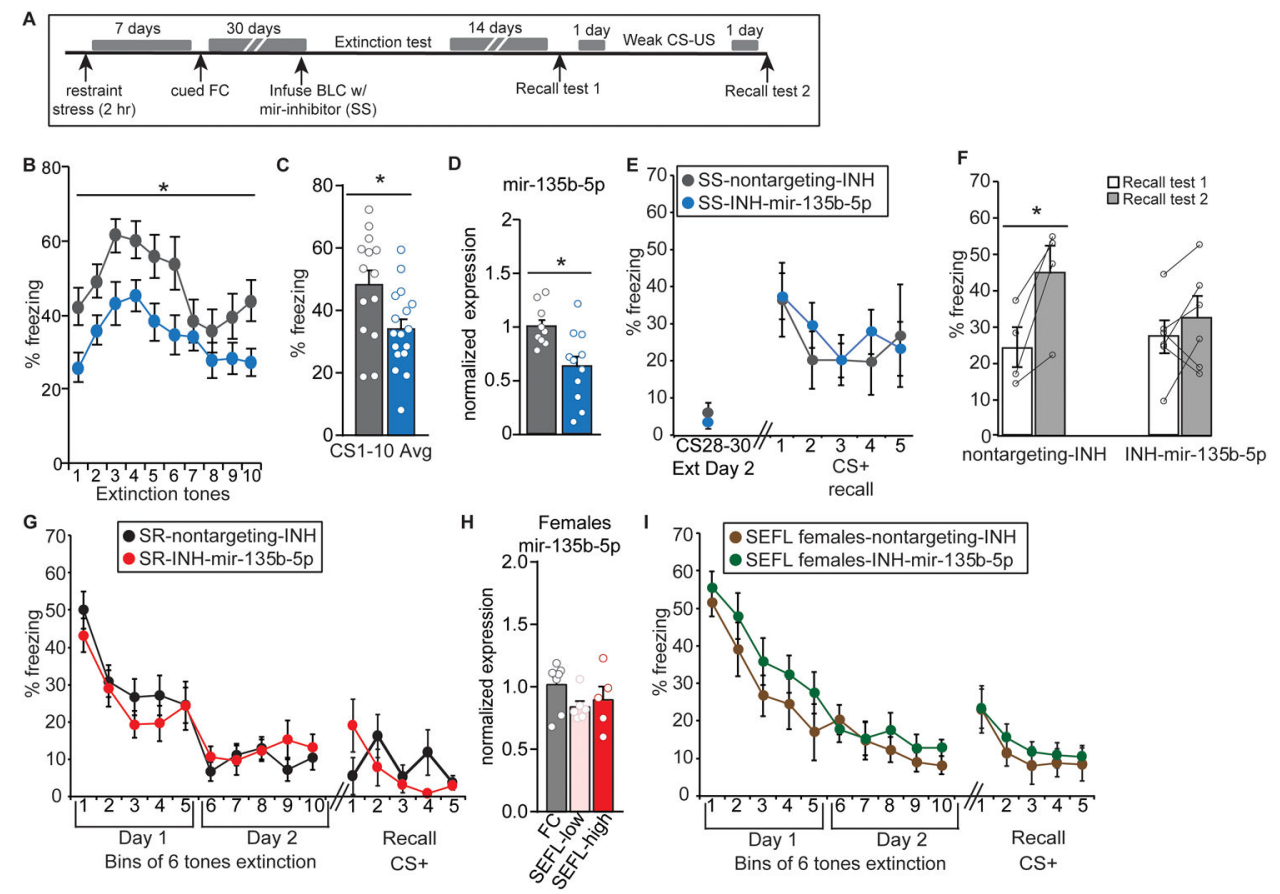

Figure 3. BLC inhibition of mir-135b-5p in SS mice weakens stress-enhanced memory expression.

(A) Overview of strategy to decrease BLC mir-135b-5p tone in SS animals. (B-C) Freezing during first 10 tones on Day 1 of extinction. RM-ANOVA for 10 tones extinction day 1: $\mathrm{F}_{(1,28)}=5.814 ; \mathrm{p}=0.023$. Unpaired $\mathrm{t}$-test for average of 10 tones: $\mathrm{t}_{(29)}=2.638, \mathrm{p}=0.0133$. Nontargeting control $n=14$, INH-mir-135b-5p $n=17$. (D) mir-135b-5p levels in SS mice after inhibition. Unpaired t-test: $\mathrm{t}_{(18)}=2.987, \mathrm{p}=0.0079$. Nontargeting control $\mathrm{n}=9$; INHmir-135b-5p $n=11$. (E) Extinction retention from the end of extinction Day 2 to the first 5 tone recall test. (F) Freezing after subthreshold re-training. Paired t-test SSnontargetingINH: $\mathrm{t}_{(3)}=3.927, \mathrm{p}=0.0294$. Nontargeting control $\mathrm{n}=4$; INH-mir-135b-5p $\mathrm{n}=$ 6. (G) Extinction (6 tone bins) for SR male mice injected with INH-mir-135b-5p or nontargeting control. (H) mir-135b-5p expression in the BLC of SEFL females (no extinction) 30 days after training. (I) Extinction (6 tone bins) for stressed female mice injected with INH-mir-135b-5p inhibitor or nontargeting control. Error bars are \pm S.E.M. 


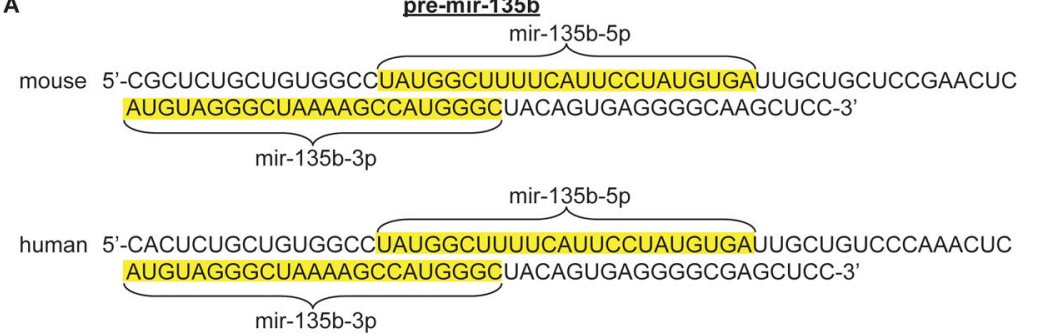

C

\begin{tabular}{llll}
\hline & $\begin{array}{l}\text { Susceptible } \\
(\mathrm{N}=8)\end{array}$ & $\begin{array}{l}\text { Resilient } \\
(\mathrm{N}=8)\end{array}$ & $\begin{array}{l}\text { Control } \\
(\mathrm{N}=8)\end{array}$ \\
\hline Post-deployment PTSD symptoms & High & Low & Low \\
Combat trauma exposure & High & High & Low \\
Age when deployed & $22.13(1.73)$ & $32.75(11.38)$ & $26.75(7.66)$ \\
BMI when deployed & $24.62(1.45)$ & $27.38(3.85)$ & $23.73(1.60)$ \\
Number of previous deployments & $0.93(1.3)$ & $1.23(1.43)$ & $0.63(0.85)$ \\
Military rank & $1.7(0.99)$ & $1.84(1.13)$ & $2.06(1.09)$ \\
Trauma exposure-score & $6.93(2.96)$ & $8.68(2.26)$ & $0.43(0.5)$ \\
Change in PTSD symptoms & $27.38(8.73)$ & $-4.125(1.24)$ & $-4.63(3.58)$ \\
\hline
\end{tabular}

B mir-135b human amygdala

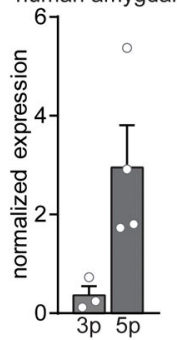

D

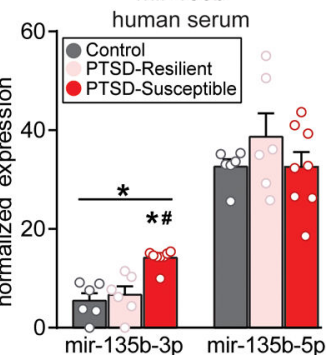

Figure 4. mir-135b is highly conserved in humans and elevated in the serum of PTSD-susceptible subjects.

(A) Conservation of the mouse pre-mir-135b sequence from mouse to human. The sequences of the 5' and 3' arms are highlighted in yellow. (B) qPCR expression of mir-135b isoforms in human amygdala, $n=3-4$ brains. (C) Characteristics of human subjects used for the evaluation of circulating mir-135b in serum. (D) Sequencing expression of mir-135b isoforms in the serum of human subjects. One-way ANOVA for mir-135b-3p: $F_{(2,17)}=$ 15.32; $\mathrm{p}=0.0002$; Post-hoc Tukey test $\mathrm{p}<0.05$ for control vs susceptible $\left(^{*}\right)$ and resilient vs susceptible (\#), $n=6-8$ /group. Error bars are \pm S.E.M. 


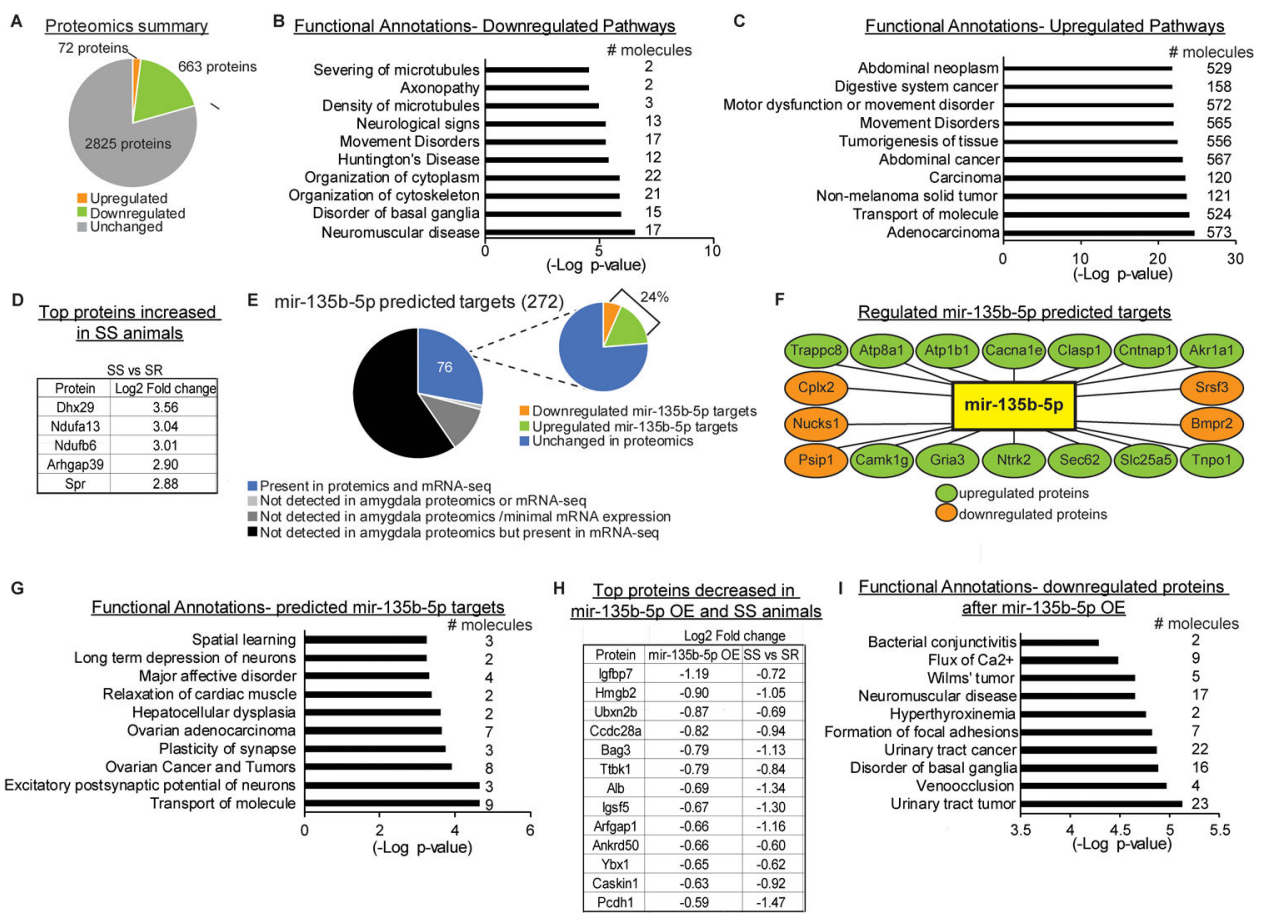

Figure 5. Global proteomic profile identifies putative mir-135b-5p targets downregulated in SS animals.

(A) Summary of the protein changes identified in BLC tissue that are at least $1.5 \log 2$ fold change between SS and SR animals 30 days after SEFL and the top 10 significant pathways of downregulated (B) or upregulated proteins (C). (D) The top 5 upregulated proteins in SS vs SR animals that were identified in the proteomics screen. (E-F) Protein expression of putative mir-135b-5p targets obtained by mass spectrometry that are $1.5 \log 2$ fold change or greater between SS and SR animals 30 days after SEFL and the top 10 significant pathways of regulated mir-135b-5p target proteins (G). (H-I) Proteins changed by at least 50\% in BLC tissue after viral mediated mir-135b-5p overexpression or SEFL and the top regulated pathways in proteins changed by at least $25 \%$ in those groups. 